\title{
Impact of donor age on liver transplant outcomes in patients with hepatocellular carcinoma: analysis of the SRTR database
}

Jie Zhou ${ }^{1,2,3,4}$, Zhichao Huang ${ }^{1}$, Zheng Chen ${ }^{1}$, Fangshen $\mathrm{Xu}^{1}$, Rongliang Tong ${ }^{1,2,3,4}$ and Shusen Zheng ${ }^{1,2,3,4^{*}}$

\begin{abstract}
Background: Donor age is an important predictor for liver transplant recipients. Studies have not fully explored its impact on transplant outcomes in hepatocellular carcinoma (HCC) patients as well as its involvement in tumor recurrence.
\end{abstract}

Methods: HCC patients who received liver transplants during 2010-2017 from the Scientific Registry of Transplant Recipients database were included. The recipients were divided into four groups based on donor age: I ( $\leq 34$ years), II (35-49 years), III (50-64 years), and IV ( $\geq 65$ years). Transplant outcomes, including the overall survival (OS), tumor recurrence, and risks, were evaluated.

Results: A total of $13,276 \mathrm{HCC}$ recipients were included in this study. Statistical significant differences were observed in OS among the four groups. The best 5-year survival was 76.0\% in group I, followed by $73.5 \%$ in group II, $72.8 \%$ in group III, and 69.2\% in group IV $(P<0.001)$. However, the liver-specific survival did not differ among these groups $(P=0.260)$. Donor age was found to be the independent predictor of OS after adjusting for other variables $(P<0.001$, ref. group I; $1.087(0.979-1.208)$ for group II, $P=0.119 ; 1.124$ (1.015-1.246) for group III, $P=0.025 ; 1.395$ (1.215-1.602) for group IV, $P<0.001$ ). In subgroup analysis, OS was significantly different in recipients with hepatitis $C$ virus (HCV), but there was no significant difference for recipients with hepatitis B virus (HBV), alcoholic liver diseases and nonalcoholic steatohepatitis (NASH). The post-transplant cumulative tumor recurrence rates were similar among the four groups $(P=0.382)$.

Conclusions: Older donor age was associated with decreased OS but not liver-specific survival as well as post-transplant tumor recurrence in HCC recipients. Donor age also had different effects in patients with different underlying liver diseases.

Keywords: Liver transplantation, Donor age, Hepatocellular carcinoma, Outcome, SRTR

\section{Background}

Hepatocellular carcinoma (HCC), the fourth most common cause of cancer-related death worldwide, accounts for more than 700,000 deaths annually and its incidence

\footnotetext{
*Correspondence: shusenzheng@zju.edu.cn

${ }^{1}$ Division of Hepatobiliary and Pancreatic Surgery, Department of Surgery, First Affiliated Hospital, Zhejiang University, School of Medicine, Hangzhou, China

Full list of author information is available at the end of the article
}

continues to increase [1-3]. Only a minority of HCC patients are feasible candidates for hepatectomy, which is due to the fact that most patients are diagnosed at the advanced stages and often presented with poor liver function. Tumor recurrence after hepatectomy also remains high. Liver transplantation is the only curative option to treat HCC patients. However, due to the disparity between the large number of candidates and the relative shortage of donor livers, many patients 
dropped out of the waiting list before they could match with a feasible donor graft.

Marginal donor usage has been suggested to expand the donor pool. Older donors are the most commonly used marginal donors. The number of older liver donors is increasing due to the ageing population [4]. However, there are concerns regarding the application of old liver grafts in clinical practice, as liver tissue from older donors would undergo a series of morphological and physiological changes, making it more vulnerable to ischemia-reperfusion injury (IRI) during transplantation [5]. These pathophysiological changes would lead to adverse effects on recipient prognosis. Feng et al. evaluated the effects of donor-related variables on transplant outcomes and demonstrated that donor age was an independent predictor of overall survival (OS) [6]. And they have created a model known as the donor risk index (DRI) to stratify the risk of transplant outcomes based on a series of donor variables. However, with an increasing application of liver grafts from older donors and improved surgical techniques as well as perioperative managements, a number of studies have recently shown the safety of use of old donor livers, even using livers from octogenarian donors [4, 7].

Despite this, the effect of donor age on liver transplantation specifically in HCC patients has not yet been fully elucidated. Tumor recurrence after liver transplantation is an important concern in those patients. We have already established recipient selection criteria so that donors are matched with the most feasible patients to obtain favorable post-transplant outcomes $[8,9]$. However, current practical experience with regard to how donor variables such as donor age could affect transplant outcomes, including tumor recurrence, in HCC patients is limited. Although previous studies have demonstrated the association between donation after cardiac death (DCD) and post-transplant mortality in HCC recipients, they have not focused on tumor recurrence [10]. Orci et al. has previously evaluated the effect of donor characteristics on tumor recurrence after liver transplantation based on recipients through 2004 to 2011 from the Scientific Registry of Transplant Recipients (SRTR) database [11]. Yet policies for treatment of HCC have changed during this time, with improvements in liver transplantation and increased use of marginal donors. Therefore, it is important to re-evaluate the donor characteristics on transplant outcomes in HCC patients in this setting.

Here, we use renewed data from the SRTR database to evaluate the effect of donor age on liver transplant outcomes, especially OS and tumor recurrence, in patients with HCC.

\section{Methods}

This study used data from the Scientific Registry of Transplant Recipients (SRTR). The SRTR data system includes data on all donor, wait-listed candidates, and transplant recipients in the US, submitted by the members of the Organ Procurement and Transplantation Network (OPTN). The Health Resources and Services Administration (HRSA), U.S. Department of Health and Human Services provides oversight to the activities of the OPTN and SRTR contractors. The data reported here have been supplied by the Hennepin Healthcare Research Institute (HHRI) as the contractor for the Scientific Registry of Transplant Recipients (SRTR). The interpretation and reporting of these data are the responsibility of the author(s) and in no way should be seen as an official policy of or interpretation by the SRTR or the U.S. Government [12]. Organs from executed prisoners were not used in this study. The protocol for the present study was in accordance with the Declaration of Helsinki and was approved by the Ethics Committee of the First Affiliated Hospital, College of Medicine, Zhejiang University, China (approval number 2019-1020).

We included patients with HCC who received liver transplantation from January 1, 2010, to December 31, 2017. The inclusion criteria were: recipients $\geq 18$ years old, with a primary diagnosis of "hepatocellular carcinoma" or "hepatoma" at transplant. Patients with a previous liver transplantation, those who received transplant for benign liver disease or liver tumor other than HCC, and those $<18$ years old were excluded from this study. Finally a total of 13,276 recipients were included in the current study. Patients were followed up to death or the end of the study on 1st March, 2019. Figure 1 illustrates the patient selection flowchart.

To assess how donor age affects transplant outcomes, we divided recipients into four groups according to donor age: group I, donor age $\leq 34$ years $(\mathrm{N}=4723)$; group II, donor age $35-49$ years $(\mathrm{N}=3572)$; group III, donor age $50-64$ years $(\mathrm{N}=3743)$; group IV, donor age $\geq 65$ years $(\mathrm{N}=1238)$. The donor and recipient data as well as tumor characteristics were compared among the four groups.

For long-term outcomes, we first compared posttransplant OS among four groups, which was evaluated from the time of LT and defined recipient death as the endpoint. OS was also assessed based on recipient underlying liver diseases including hepatitis $\mathrm{C}$ virus $(\mathrm{HCV})$, hepatitis $B$ virus (HBV), alcoholic liver diseases and nonalcoholic steatohepatitis (NASH). Liver-specific survival was also analyzed, which was measured from the date of LT to date of liver-related death or last follow-up. Patient cause of death, including graft failure, cardiovascular/cerebrovascular disease, organ failure, hemorrhage, infection and HCC recurrence, were compared among 


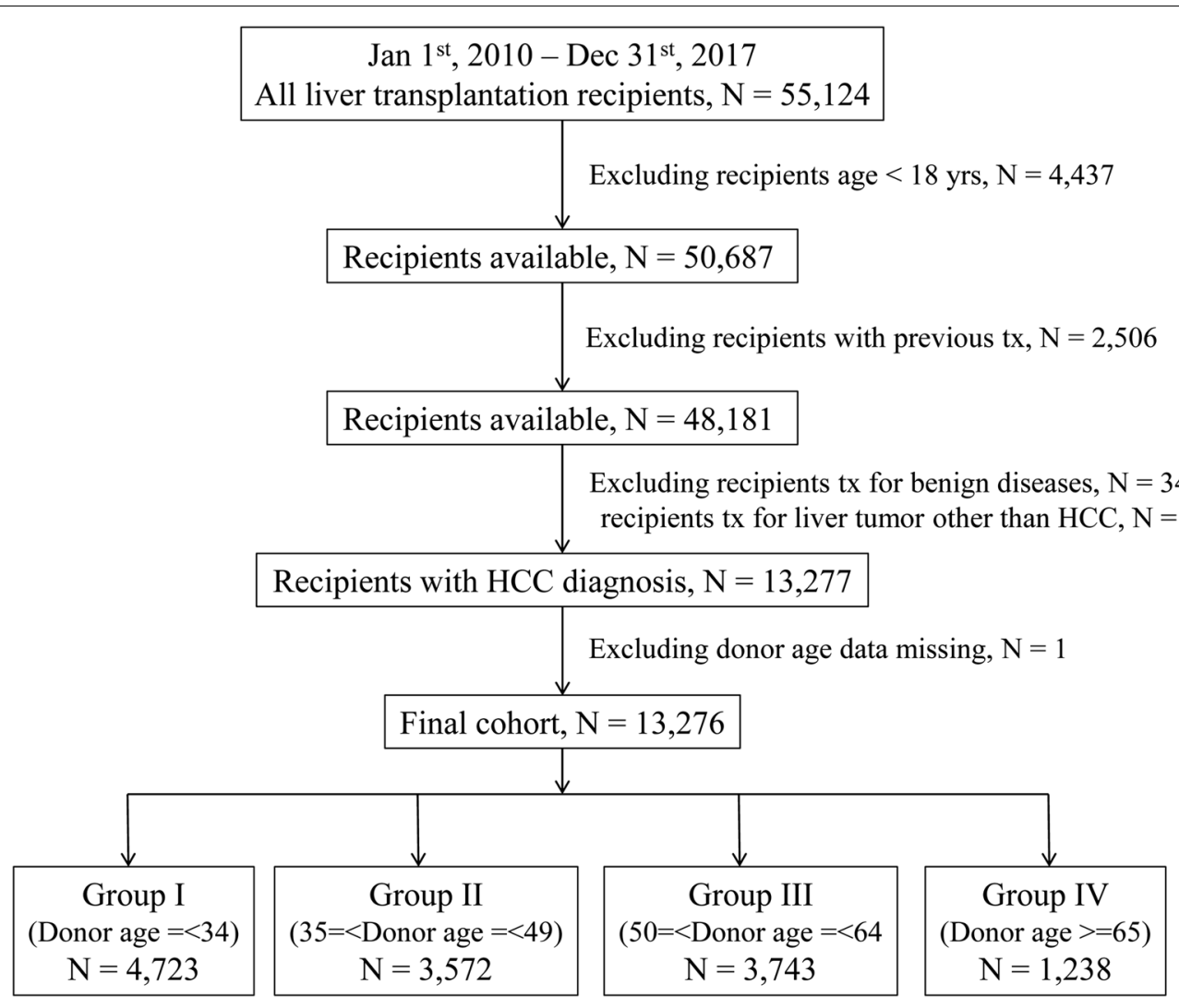

Fig. 1 Flow chart of the patient selection process

the four groups, first within 30 days after transplantation and then for the whole population. The post-transplant $\mathrm{HCC}$ recurrence was compared among the four groups, and the definition was in accordance with the report by Samoylova et al. [13]. The detailed identification procedure was performed in accordance with that described in the study by Orci et al. [11].

\section{Statistical analysis}

Baseline characteristics were compared using one-way ANOVA for continuous variables and the chi-square test for binomial variables. OS and liver-specific survival was assessed by the Kaplan-Meier method and log-rank test was used to compare differences among the groups. Univariate analysis was used to identify potential predictors for OS, and those with $P<0.05$ were further analyzed in the multivariable analysis using the Cox proportional hazard ratios (HRs) model. Time-dependent effects were evaluated based on Schoenfeld's residuals, and cubic spline functions were introduced in the model [14]. The cumulative tumor recurrence rates were evaluated using the competing risk model. A two-tailed P value $<0.05$ was considered statistically significant. All the analyses were conducted with SPSS version 22.0 (IBM, Armonk, NY, United States) and R for Windows (version 4.0.2).

\section{Results}

\section{Baseline characteristics}

The median follow-up time was 36 months (interquartile range, 18-60 months) for the entire study population. Donor characteristics including donor height, weight, race, ABO blood type, sex, cause of death, deceased donor type (DCD or not) significantly differed among the four groups. For recipient characteristics, the recipient age, race, $\mathrm{ABO}$ blood type, underlying liver disease, height, weight, cold ischemia time, body mass index (BMI), laboratory model for end-stage liver disease (MELD) score, albumin, bilirubin, international normalized ratio (INR), creatinine and immunosuppression maintenance with tacrolimus, cyclosporin, mycophenolate mofetil and steroids at discharge were all statistically different among the four groups. However, the recipient warm ischemia time, pre-transplant sodium level and immunosuppression induction (with anti-CD25 or thymoglobulin) as well as sirolimus maintenance at discharge were comparable among the four groups. Analysis of tumor characteristics revealed that the pre-transplant 
treatment (including transarterial chemoembolization (TACE), radiofrequency ablation (RFA), chemotherapy, cryoablation, surgery), number of tumors, largest tumor diameter, sum of tumor diameters, tumor staging, and pre-transplant $\alpha$-fetoprotein (AFP) level were all similar among four groups. Table 1 presents a summary of the data in detail.

OS

We compared OS among the four groups. The 1-, 3-, and 5 -year OS were $91.4 \%, 82.3 \%$, and $76.0 \%$ in group I; $90.6 \%, 81.2 \%$, and $73.5 \%$ in group II; $89.5 \%, 80.0 \%$, and $72.8 \%$ in group III; and $89.2 \%, 76.8 \%$, and $69.2 \%$ in group IV, respectively $(P<0.001$; Fig. 2$)$. We also compared the OS between two groups at a time, and observed that the survival of group I recipients was better than that of the other three groups (group I vs. group II, $P=0.049$; group I vs. group III, $P=0.002$; group I vs. group IV, $P<0.001$ ). The survival of group II recipients was comparable to that of group III $(P=0.308)$ but significantly better than that of group IV recipients $(P=0.004)$. The survival of group III recipients was also better than group IV $(P=0.038)$. We also compared OS after excluding patients whose follow-up time was $<24$ months, and observed similar outcomes (Additional file 1: Table S1 and Additional file 2: Figure S1). For liver-specific survival, no differences were observed among four age groups (Additional file 1: Table S2 and Additional file 3: Figure S2).

No differences were observed for cause of death within post-transplant 30 days. However, recipients had higher incidence rates of graft failure $(P=0.009)$, organ failure $(P=0.002)$ and infection $(P=0.001)$ in the older donor age group during follow-up (Table 2).

Next, we analyzed the survival according to recipient underlying liver diseases. The OS significantly differed in recipients with $\mathrm{HCV}$, with 1-, 3-, and 5-year OS rates at $91.4 \%, 82.1 \%$, and $75.8 \%$ in group I; $90.2 \%, 79.8 \%$, and $72.1 \%$ in group II; $89.1 \%, 78.6 \%$, and $71.4 \%$ in group III; and $89.3 \%, 75.1 \%$, and $68.1 \%$ in group IV, respectively $(P<0.001$; Fig. 3a). However, there were no significant differences in the survival rates among the four groups of patients in subsets with $\mathrm{HBV}$, alcoholic liver diseases and NASH. The 1-, 3-, and 5-year OS rates were 93.3\%, $87.5 \%$, and $84.1 \%$ in group I; $90.3 \%, 85.2 \%$, and $80.6 \%$ in group II; $91.5 \%, 85.0 \%$, and $82.0 \%$ in group III; and $92.1 \%$, $83.1 \%$, and $77.1 \%$ in group IV in recipients with HBV $(P=0.564$; Fig. $3 \mathrm{~b})$. For recipients with alcoholic liver diseases, the 1-, 3-, and 5-year OS rates were $89.7 \%, 81.0 \%$, and $75.4 \%$ in group I; $92.4 \%, 84.1 \%$, and $76.0 \%$ in group II; $91.2 \%, 83.0 \%$, and $75.8 \%$ in group III; and $90.2 \%, 79.4 \%$, and $71.2 \%$ in group IV $(P=0.850$; Fig. $3 \mathrm{c})$. The OS rates in recipients with NASH were $88.3 \%, 80.8 \%$, and $73.9 \%$ in group I; $89.0 \%, 82.7 \%$, and $72.9 \%$ in group II; $90.0 \%$,
$83.0 \%$, and $76.4 \%$ in group III; and $83.5 \%, 76.3 \%$, and $68.2 \%$ in group IV $(P=0.442$; Fig. $3 d)$. OS rates were also analyzed after excluding patients whose follow-up time was $<24$ months, and similar outcomes were observed (Additional file 1: Table S3 and Additional file 4: Figure S3). Liver-specific survival was also analyzed and there were no differences among four groups in underlying liver diseases (Additional file 1: Table S4 and Additional file 5: Figure S4).

\section{Univariate analysis for OS}

We then performed univariate analysis to identify potential risk factors for recipient OS. Donor characteristics, including donor age and cause of death; recipient characteristics, including recipient age, race, underlying liver diseases, pre-transplant laboratory MELD score, albumin, bilirubin, INR, creatinine, and sodium levels; tumor characteristics including number of tumors, largest tumor diameter, sum of tumor diameters, tumor staging, and pre-transplant AFP level; immunosuppression maintenance with tacrolimus, mycophenolate mofetil and steroid at discharge were all found to be significantly associated with the OS. Table 3 presents this information in greater detail.

\section{Multivariable analysis for $\mathrm{OS}$}

The multivariable Cox regression analysis showed that donor age, recipient age, race, underlying liver diseases, pre-transplant MELD score, creatinine, sum of tumor diameters, AFP level, immunosuppression maintenance with tacrolimus and steroid at discharge were all independent predictors of OS. Table 4 presents this information in greater detail. Further univariate as well as multivariable analysis for OS were also performed for those patients with follow-up time $\geq 24$ months and detailed information was shown in Additional file 1: Table S5 and S6.

\section{Time-dependent effect of donor age on OS}

We specifically analyzed the time-dependent HR of donor age on the recipient OS and observed that the $\mathrm{HR}$ remained generally stable during the follow-up, but it tended to increase in the early transplant months and decrease over the years (Fig. 4). This suggested that although donor age was a constant independent predictor of decreased OS, the negative effect of older donor age may tend to decrease over time.

\section{Post-transplant tumor recurrence}

A total of 567 recipients suffered HCC recurrence in the post-transplant period. Patients with tumor recurrence had significantly inferior OS compared to those without 
Table 1 Baseline characteristics

\begin{tabular}{|c|c|c|c|c|c|}
\hline & $\begin{array}{l}\text { Group I } \\
\text { (Donor age } \leq 34 \text {, } \\
\mathrm{N}=4723 \text { ) }\end{array}$ & $\begin{array}{l}\text { Group II } \\
\text { (Donor age 35-49, } \\
\mathrm{N}=3572 \text { ) }\end{array}$ & $\begin{array}{l}\text { Group III } \\
\text { (Donor age 50-64, } \\
\mathrm{N}=3743 \text { ) }\end{array}$ & $\begin{array}{l}\text { Group IV } \\
\text { (Donor age } \geq 65 \text {, } \\
N=1238 \text { ) }\end{array}$ & $P$ value \\
\hline \multicolumn{6}{|l|}{ Donorvariables } \\
\hline Race & & & & & $<0.001$ \\
\hline White & $3051(64.6 \%)$ & $2269(63.5 \%)$ & $2378(63.5 \%)$ & $872(70.4 \%)$ & \\
\hline Black or African American & $814(17.2 \%)$ & $647(18.1 \%)$ & $757(20.2 \%)$ & $188(15.2 \%)$ & \\
\hline Asian & $82(1.7 \%)$ & $96(2.7 \%)$ & $114(3.0 \%)$ & $51(4.1 \%)$ & \\
\hline Hispanic/Latino & $725(15.4 \%)$ & $524(14.7 \%)$ & $465(12.4 \%)$ & $123(9.9 \%)$ & \\
\hline Other & $51(1.1 \%)$ & $36(1.0 \%)$ & $29(0.8 \%)$ & $4(0.3 \%)$ & \\
\hline $\mathrm{ABO}$ & & & & & $<0.001$ \\
\hline A & $1752(37.1 \%)$ & 1361 (38.1\%) & $1346(36.0 \%)$ & 467 (37.7\%) & \\
\hline B & $667(14.1 \%)$ & $490(13.7 \%)$ & $506(13.5 \%)$ & $129(10.4 \%)$ & \\
\hline $\mathrm{O}$ & $2129(45.1 \%)$ & $1584(44.3 \%)$ & $1796(48.0 \%)$ & $620(50.1 \%)$ & \\
\hline$A B$ & $175(3.7 \%)$ & $137(3.8 \%)$ & $95(2.5 \%)$ & $22(1.8 \%)$ & \\
\hline Gender & & & & & $<0.001$ \\
\hline M & $3226(68.3 \%)$ & $2048(57.3 \%)$ & $2013(53.8 \%)$ & $619(50.0 \%)$ & \\
\hline $\mathrm{F}$ & 1497 (31.7\%) & $1524(42.7 \%)$ & $1730(46.2 \%)$ & $619(50.0 \%)$ & \\
\hline Cause of death & & & & & $<0.001$ \\
\hline Anoxia & $1812(39.4 \%)$ & $1262(36.5 \%)$ & $1045(28.3 \%)$ & $202(16.3 \%)$ & \\
\hline Cerebrovascular/stroke & $429(9.3 \%)$ & $1285(37.1 \%)$ & $2003(54.2 \%)$ & $815(65.9 \%)$ & \\
\hline Head trauma & $2234(48.6 \%)$ & $801(23.1 \%)$ & $586(15.8 \%)$ & $205(16.6 \%)$ & \\
\hline CNS tumor & $27(0.6 \%)$ & $25(0.7 \%)$ & $12(0.3 \%)$ & $1(0.1 \%)$ & \\
\hline Other & $94(2.0 \%)$ & $88(2.5 \%)$ & $52(1.4 \%)$ & $14(1.1 \%)$ & \\
\hline $\mathrm{DCD}$ & $494(10.5 \%)$ & $302(8.5 \%)$ & $153(4.1 \%)$ & $1(0.1 \%)$ & $<0.001$ \\
\hline Height (cm) & $172.58 \pm 11.56$ & $171.48 \pm 10.11$ & $170.32 \pm 10.13$ & $168.83 \pm 10.18$ & $<0.001$ \\
\hline Weight (kg) & $78.71 \pm 20.49$ & $86.30 \pm 21.54$ & $84.02 \pm 20.75$ & $79.67 \pm 18.30$ & $<0.001$ \\
\hline \multicolumn{6}{|l|}{ Recipient variables } \\
\hline Gender & & & & & $<0.001$ \\
\hline M & $3599(76.2 \%)$ & $2854(79.9 \%)$ & $2929(78.3 \%)$ & 909 (73.4\%) & \\
\hline $\mathrm{F}$ & $1124(23.8 \%)$ & $718(20.1 \%)$ & $814(21.7 \%)$ & $329(26.6 \%)$ & \\
\hline Age & $59.62 \pm 7.31$ & $59.49 \pm 7.16$ & $59.89 \pm 6.75$ & $61.55 \pm 6.64$ & $<0.001$ \\
\hline Race & & & & & $<0.001$ \\
\hline White & $3074(65.1 \%)$ & $2400(67.2 \%)$ & $2524(67.4 \%)$ & $825(66.6 \%)$ & \\
\hline Black or African American & $500(10.6 \%)$ & $373(10.4 \%)$ & $332(8.9 \%)$ & $85(6.9 \%)$ & \\
\hline Asian & $332(7.0 \%)$ & $203(5.7 \%)$ & $253(6.8 \%)$ & $109(8.8 \%)$ & \\
\hline Hispanic/Latino & $753(15.9 \%)$ & $552(15.5 \%)$ & $590(15.8 \%)$ & $201(16.2 \%)$ & \\
\hline Other & $64(1.4 \%)$ & $44(1.2 \%)$ & $44(1.2 \%)$ & $18(1.5 \%)$ & \\
\hline $\mathrm{ABO}$ & & & & & $<0.001$ \\
\hline A & 1733 (36.7\%) & $1344(37.6 \%)$ & $1328(35.5 \%)$ & $462(37.3 \%)$ & \\
\hline B & $684(14.5 \%)$ & 509 (14.2\%) & $498(13.3 \%)$ & $131(10.6 \%)$ & \\
\hline $\mathrm{O}$ & $2062(43.7 \%)$ & $1532(42.9 \%)$ & $1780(47.6 \%)$ & $609(49.2 \%)$ & \\
\hline$A B$ & $244(5.2 \%)$ & $187(5.2 \%)$ & $137(3.7 \%)$ & $36(2.9 \%)$ & \\
\hline Underlying liver disease & & & & & $<0.001$ \\
\hline $\mathrm{HCV}$ & $3147(66.6 \%)$ & $2389(66.9 \%)$ & $2372(63.4 \%)$ & $556(44.9 \%)$ & \\
\hline HBV & $329(7.0 \%)$ & $228(6.4 \%)$ & $271(7.2 \%)$ & $152(12.3 \%)$ & \\
\hline Alcohol & $412(8.7 \%)$ & $347(9.7 \%)$ & $366(9.8 \%)$ & $184(14.9 \%)$ & \\
\hline NASH & $396(8.4 \%)$ & $309(8.7 \%)$ & $363(9.7 \%)$ & $171(13.8 \%)$ & \\
\hline Other & $439(9.3 \%)$ & $299(8.4 \%)$ & $371(9.9 \%)$ & $175(14.1 \%)$ & \\
\hline Height (cm) & $172.39 \pm 10.04$ & $173.06 \pm 9.85$ & $172.75 \pm 9.99$ & $170.91 \pm 9.96$ & $<0.001$ \\
\hline Weight (kg) & $85.07 \pm 18.82$ & $86.72 \pm 18.52$ & $86.75 \pm 18.66$ & $84.40 \pm 17.84$ & $<0.001$ \\
\hline
\end{tabular}


Table 1 (continued)

\begin{tabular}{|c|c|c|c|c|c|}
\hline & $\begin{array}{l}\text { Group I } \\
\text { (Donor age } \leq 34 \text {, } \\
\mathrm{N}=4723 \text { ) }\end{array}$ & $\begin{array}{l}\text { Group II } \\
\text { (Donor age 35-49, } \\
\mathrm{N}=3572 \text { ) }\end{array}$ & $\begin{array}{l}\text { Group III } \\
\text { (Donor age 50-64, } \\
\mathrm{N}=3743 \text { ) }\end{array}$ & $\begin{array}{l}\text { Group IV } \\
\text { (Donor age } \geq 65 \text {, } \\
N=1238 \text { ) }\end{array}$ & $P$ value \\
\hline Warm ischemia time (min) & $40.11 \pm 20.31$ & $40.29 \pm 18.80$ & $40.86 \pm 19.69$ & $40.61 \pm 21.13$ & 0.646 \\
\hline Cold ischemia time (h) & $6.21 \pm 2.65$ & $6.06 \pm 2.52$ & $6.21 \pm 2.50$ & $6.02 \pm 2.21$ & 0.006 \\
\hline BMI & $28.55 \pm 5.47$ & $29.00 \pm 7.73$ & $29.04 \pm 6.24$ & $28.83 \pm 5.40$ & 0.001 \\
\hline MELD & $15.40 \pm 8.77$ & $15.40 \pm 8.53$ & $14.74 \pm 7.97$ & $14.01 \pm 6.83$ & $<0.001$ \\
\hline Albumin (g/dl) & $3.21 \pm 0.69$ & $3.23 \pm 0.69$ & $3.25 \pm 0.70$ & $3.27 \pm 0.67$ & 0.01 \\
\hline Bilirubin (mg/dl) & $4.05 \pm 7.32$ & $3.91 \pm 7.01$ & $3.56 \pm 6.39$ & $3.21 \pm 5.62$ & $<0.001$ \\
\hline INR & $1.53 \pm 0.73$ & $1.55 \pm 0.79$ & $1.51 \pm 0.74$ & $1.46 \pm 0.68$ & 0.002 \\
\hline Creatinine (mg/dl) & $1.23 \pm 1.13$ & $1.19 \pm 1.01$ & $1.12 \pm 0.86$ & $1.04 \pm 0.62$ & $<0.001$ \\
\hline Sodium (mmol/L) & $137.12 \pm 4.51$ & $137.16 \pm 4.42$ & $137.28 \pm 4.40$ & $137.17 \pm 4.45$ & 0.389 \\
\hline \multicolumn{6}{|l|}{ Treatment } \\
\hline TACE & 2577 (64.9\%) & 1990 (65.5\%) & $2140(65.6 \%)$ & $757(69.0 \%)$ & 0.093 \\
\hline RFA & $489(12.3 \%)$ & $341(11.2 \%)$ & $365(11.2 \%)$ & $108(9.8 \%)$ & 0.107 \\
\hline Chemotherapy & $123(3.1 \%)$ & $89(2.9 \%)$ & $116(3.6 \%)$ & $32(2.9 \%)$ & 0.482 \\
\hline Cryoablation & $12(0.3 \%)$ & $13(0.4 \%)$ & $14(0.4 \%)$ & $2(0.2)$ & 0.54 \\
\hline Surgery & $39(1.0 \%)$ & $31(1.0 \%)$ & $43(1.3 \%)$ & $18(1.6 \%)$ & 0.207 \\
\hline Tumor staging & & & & & 0.592 \\
\hline Within Milan & $3680(97.4 \%)$ & 2795 (96.8\%) & $3026(97.1 \%)$ & $1023(97.2 \%)$ & \\
\hline Beyond Milan & $99(2.6 \%)$ & $92(3.2 \%)$ & $91(2.9 \%)$ & $30(2.8 \%)$ & \\
\hline Tumor nubmer & $1.24 \pm 0.55$ & $1.24 \pm 0.54$ & $1.24 \pm 0.56$ & $1.25 \pm 0.55$ & 0.197 \\
\hline Largest tumor diameter (cm) & $1.54 \pm 1.40$ & $1.57 \pm 1.61$ & $1.57 \pm 1.40$ & $1.57 \pm 1.44$ & 0.463 \\
\hline Sum of tumor diameters $(\mathrm{cm})$ & $1.88 \pm 1.81$ & $1.91 \pm 1.98$ & $1.91 \pm 1.80$ & $1.94 \pm 1.88$ & 0.381 \\
\hline AFP value $(\mathrm{ng} / \mathrm{ml})$ & $8(4,24)$ & $8(4,27)$ & $8(4,24)$ & $7(4,20)$ & 0.781 \\
\hline \multicolumn{6}{|l|}{ Immunosuppression } \\
\hline Induction & & & & & 0.096 \\
\hline Anti-CD25 & 826 & 599 & 633 & 211 & \\
\hline Thymoglobulin & 398 & 334 & 290 & 84 & \\
\hline Anti-CD25 + Thymoglobulin & 10 & 5 & 9 & 0 & \\
\hline \multicolumn{6}{|l|}{ Maintenance } \\
\hline Tacrolimus & 3179 & 2309 & 2392 & 751 & $<0.001$ \\
\hline Cyclosporin & 117 & 93 & 78 & 16 & 0.036 \\
\hline Sirolimus & 154 & 118 & 119 & 46 & 0.834 \\
\hline Mycophenolate mofetil & 2236 & 1733 & 1694 & 570 & 0.036 \\
\hline Steroids & 1709 & 1244 & 1248 & 459 & 0.021 \\
\hline
\end{tabular}

AFP, a-fetoprotein; BMI, body mass index; CNS, central nervous system; DCD, donation after cardiac death; HBV, hepatitis B virus; $\mathrm{HCV}$, hepatitis C virus; INR, international normalized ratio; MELD, model for end-stage liver disease; NASH, nonalcoholic steatohepatitis; RFA, radiofrequency ablation; TACE, transarterial chemoembolization

recurrence (5-year survival $8.7 \%$ vs. $77.8 \%$ respectively, $P<0.001$, Additional file 6: Figure S5).

The general tumor recurrence rates were comparable among the four age groups (218 (4.6\%) in group I, 142 (4.0\%) in group II, 150 (4.0\%) in group III and 57 (4.6\%) in group IV, $P=0.375)$. The median time to recurrence after transplantation was 22 months (interquartile range, 10-33 months) in group I, 22.5 months (12-35 months) in group II, 21.5 months (12-35.5 months) in group III and 22 months (11-37 months) in group IV. To further analyze the cumulative tumor recurrence rates, we introduced the competing risk model. We observed that the cumulative recurrence rates in the four groups were also similar, with a 5-year cumulative recurrence rate of $5.54 \%$ in group I, $4.98 \%$ in group II, $4.80 \%$ in group III and $5.84 \%$ in group IV $(P=0.382$, Additional file 7 : Figure S6).The cumulative recurrence rate after excluding patients whose follow-up time $<24$ months were also compared among four groups and showed similar trends $(P=0.368)$.

As no difference was observed in terms of recurrence rates among the groups, additional Cox regression model 


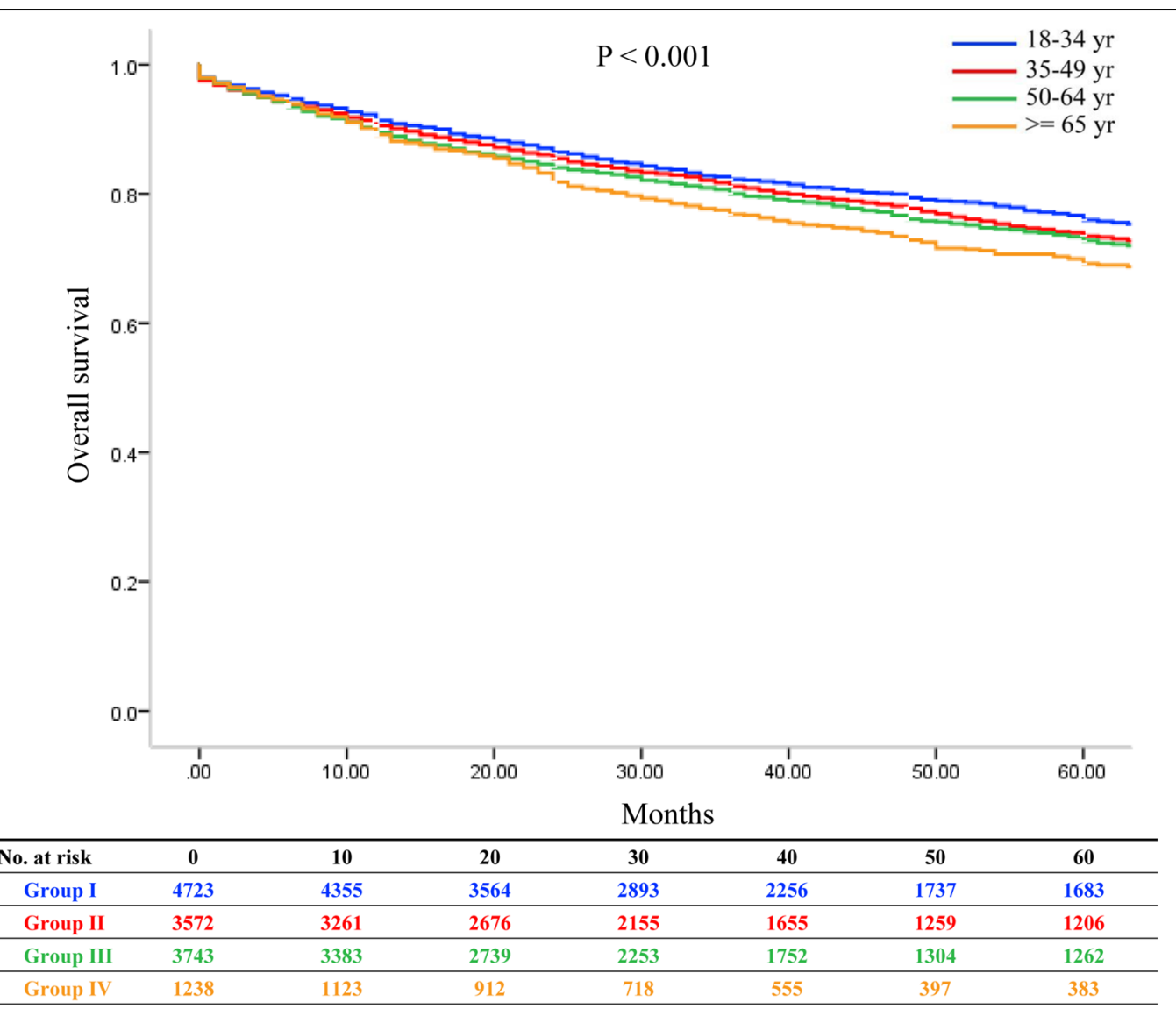

Fig. 2 Overall survival of HCC recipients categorized by different donor age groups

and competing regression model were not used. However, we further investigated the time-dependent effect of donor age on tumor recurrence in a sensitive analysis. The effect of donor age also remained generally stable in the early transplant years, and showed a decreasing trend over the long-term follow-up period (Additional file 8: Figure S7).

\section{Discussion}

In this study, we demonstrated that the OS differed in HCC liver transplant recipients categorized according to different donor ages, whereas donor age had no effect on post-transplant tumor recurrence. With regard to underlying liver diseases, there were differences in $\mathrm{OS}$ in $\mathrm{HCV}$ recipients, but not in recipients with $\mathrm{HBV}$, alcoholic liver diseases or NASH.

The impact of donor age on transplant outcomes has been evaluated for decades with controversies surrounding the safety and feasibility of grafts from older donors. Some studies revealed the association between older donor age and decreased recipient survival while others shared successful experience of the use of septuagenarian and even octogenarian donors [4, 6, 7, 15-17]. Also, little is known about how donor age affects tumor recurrence in recipients with HCC. Studies have shown that liver grafts from older donors would be more vulnerable to IRI [5]. And increased IRI was found to be associated with tumor recurrence $[18,19]$. Thus, there is a potential that older donor age can lead to higher tumor recurrence rate after transplantation.

We found that there were significant statistical differences in OS among four age groups, while no difference was observed with regard to tumor recurrence. Indeed the actual survival differences were minimal among four groups, especially at post-transplant 1 year. The significant statistical difference in OS might be due to the large cohort of patients included in this study, which might not necessarily mean clinical relevance. Moreover, the liver-specific survival was similar among four groups, which might indicate that older donor age had minimal impact on liver graft to affect long-term survival in HCC recipients.

We also observed the differential effects of donor age on recipients with different underlying liver diseases. In 


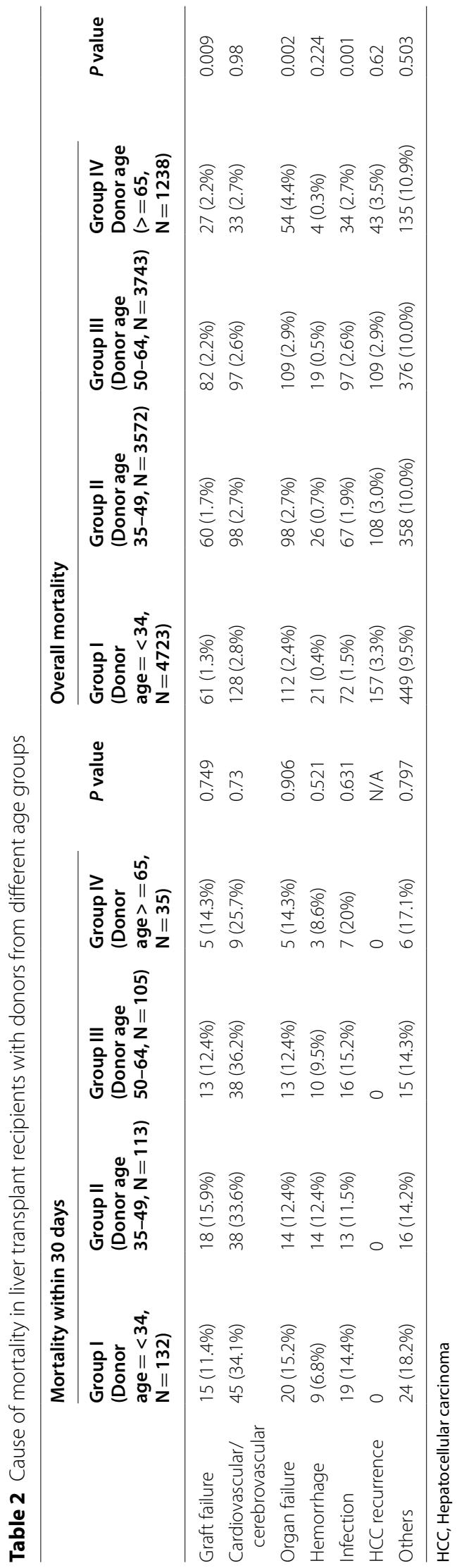



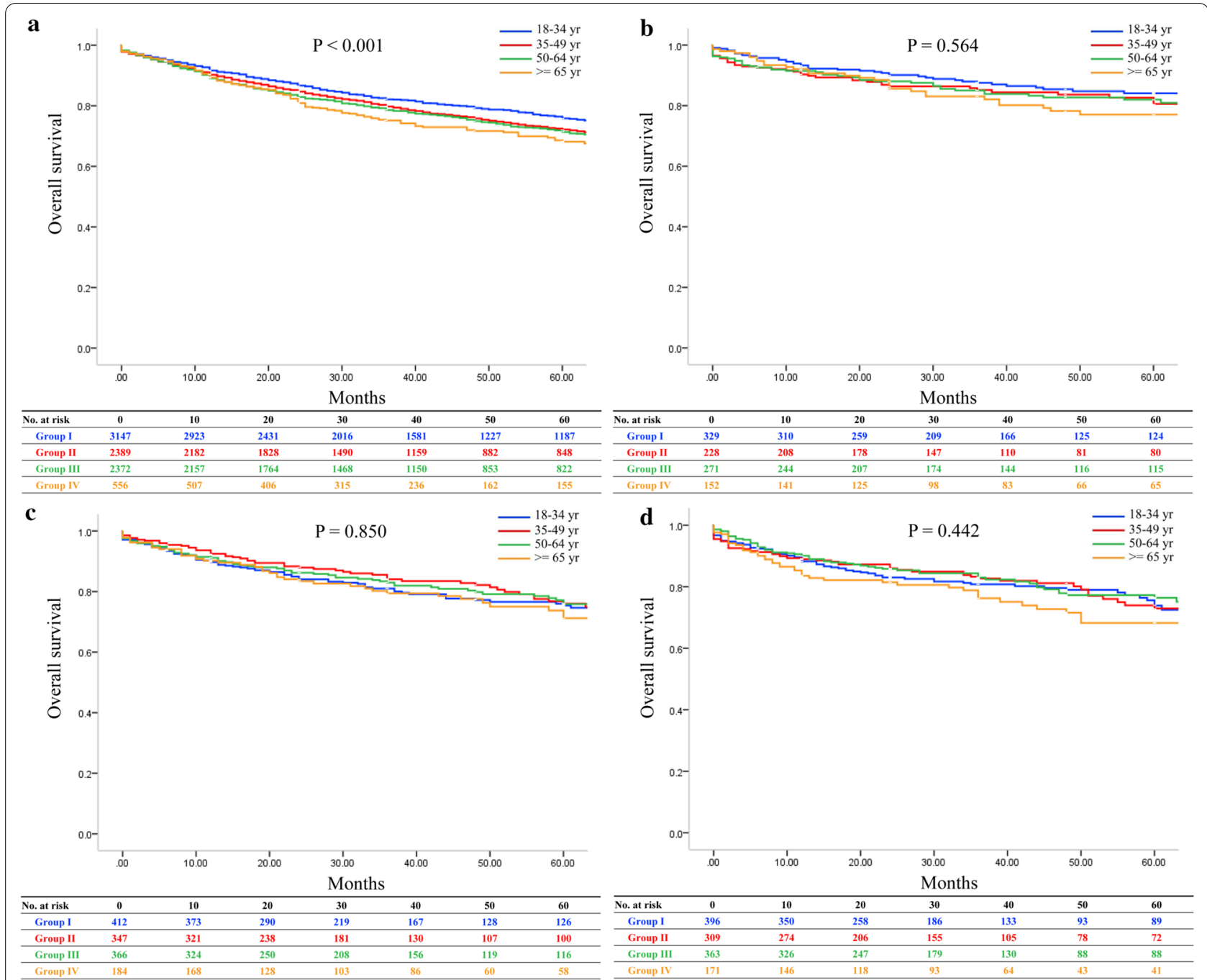

Fig. 3 Overall survival of HCC recipients in different donor age groups according to underlying liver diseases: a HCV; $\mathbf{b} \mathrm{HBV}$; $\mathbf{c}$ alcoholic liver diseases; d NASH

patients with $\mathrm{HCV}$, older donor age was associated with decreased OS, while in patients with HBV, alcoholic liver diseases and NASH, no differences in OS were observed among four groups. This result is in accordance with that reported by Lake et al. [20], who reported that donor age did not have any effect on post-transplant outcomes in recipients with HBV. Their study also showed that transplants from donors $>60$ years were associated with decreased survival in patients with underlying liver diseases other than HBV and HCV. However, they did not sub-classify these diseases, which we think is important in the current transplant practice, as the number of patients with NASH has been increasing in recent years and it has become one of the leading indications of liver transplantation [21, 22]. Therefore, our preliminary results of impact of donor age on different underlying liver diseases in HCC population is worth validation and further exploration to study the different causes of liver diseases in transplant recipients.

Recipient age was also found to be an independent predictor of OS in $\mathrm{HCC}$ recipients, which was in accordance with previous literature [23-26]. Patients with HCC are generally older than other candidates on the waiting list [22, 25, 27]. Factors including cardiovascular diseases, functional status such as frailty as well as higher extrahepatic tumor risks are all the negative factors associated with older recipient age, which may lead to adverse outcomes. Moreover, sum of tumor diameters and pretransplant AFP level were independent predictors of OS, reflecting the importance of the candidate selection 
Table 3 Univariate analysis of predictors for recipient overall survival

\begin{tabular}{|c|c|c|}
\hline & $\mathrm{HR}(95 \% \mathrm{Cl})$ & $P$ value \\
\hline \multicolumn{3}{|l|}{ Donorvariables } \\
\hline Age (reference Group I) & & $<0.001$ \\
\hline Group ॥ & $1.097(1.000-1.203)$ & 0.050 \\
\hline Group III & $1.153(1.053-1.262)$ & 0.002 \\
\hline Group IV & $1.319(1.164-1.493)$ & $<0.001$ \\
\hline Race (reference White) & & 0.778 \\
\hline Black or African American & $1.008(0.917-1.108)$ & 0.868 \\
\hline Asian & $0.983(0.783-1.235)$ & 0.885 \\
\hline Hispanic/Latino & $1.011(0.910-1.124)$ & 0.832 \\
\hline Other & $0.750(0.483-1.165)$ & 0.200 \\
\hline$A B O$ (reference $A$ ) & & 0.123 \\
\hline B & $0.925(0.824-1.039)$ & 0.189 \\
\hline O & $1.053(0.974-1.139)$ & 0.196 \\
\hline$A B$ & $0.961(0.782-1.181)$ & 0.706 \\
\hline Gender & $1.005(0.969-1.042)$ & 0.781 \\
\hline Cause of death (reference Anoxia) & & 0.002 \\
\hline Cerebrovascular/stroke & $1.112(1.019-1.214)$ & 0.017 \\
\hline Head trauma & $0.960(0.874-1.055)$ & 0.397 \\
\hline CNS tumor & $0.452(0.215-0.950)$ & 0.036 \\
\hline Other & $0.955(0.722-1.263)$ & 0.747 \\
\hline DCD & $1.052(0.916-1.208)$ & 0.476 \\
\hline Height (cm) & $0.997(0.994-1.000)$ & 0.085 \\
\hline Weight (kg) & $1.000(0.999-1.002)$ & 0.587 \\
\hline \multicolumn{3}{|l|}{ Recipient variables } \\
\hline Age & $1.018(1.013-1.024)$ & $<0.001$ \\
\hline Gender (F vs. M) & $0.972(0.931-1.015)$ & 0.195 \\
\hline Race (reference White) & & $<0.001$ \\
\hline Black or African American & $1.177(1.050-1.320)$ & 0.005 \\
\hline Asian & $0.756(0.645-0.885)$ & $<0.001$ \\
\hline Hispanic/Latino & $0.852(0.767-0.946)$ & 0.003 \\
\hline Other & $0.965(0.706-1.320)$ & 0.826 \\
\hline$A B O$ (reference $A$ ) & & 0.226 \\
\hline B & $0.946(0.844-1.061)$ & 0.345 \\
\hline O & $1.044(0.965-1.130)$ & 0.28 \\
\hline$A B$ & $0.927(0.773-1.111)$ & 0.41 \\
\hline Underlying liver disease (reference HCV) & & $<0.001$ \\
\hline HBV & $0.687(0.586-0.804)$ & $<0.001$ \\
\hline Alcohol & $0.905(0.796-1.029)$ & 0.126 \\
\hline $\mathrm{NASH}$ & $1.014(0.890-1.154)$ & 0.839 \\
\hline Other & $1.038(0.922-1.169)$ & 0.535 \\
\hline Height (cm) & $1.003(1.000-1.007)$ & 0.071 \\
\hline Weight (kg) & $1.000(0.998-1.002)$ & 0.849 \\
\hline Warm ischemia time (min) & $1.000(0.998-1.003)$ & 0.674 \\
\hline Cold ischemia time (h) & $0.999(0.986-1.013)$ & 0.915 \\
\hline BMI & $0.997(0.990-1.003)$ & 0.303 \\
\hline MELD & $1.016(1.012-1.020)$ & $<0.001$ \\
\hline Albumin (g/dl) & $0.893(0.848-0.940)$ & $<0.001$ \\
\hline Bilirubin (mg/dl) & $1.013(1.008-1.017)$ & $<0.001$ \\
\hline INR & $1.084(1.040-1.131)$ & $<0.001$ \\
\hline
\end{tabular}


Table 3 (continued)

\begin{tabular}{llr}
\hline & HR (95\% Cl) & $P$ value \\
\hline Creatinine (mg/dl) & $1.086(1.061-1.111)$ & $<0.001$ \\
Sodium (mmol/L) & $0.991(0.983-0.999)$ & 0.034 \\
Tumor staging (beyond vs. within Milan) & $1.420(1.160-1.738)$ & 0.001 \\
Tumor nubmer & $1.113(1.043-1.187)$ & 0.001 \\
Largest tumor diameter (cm) & $1.065(1.045-1.086)$ & $<0.001$ \\
Sum of tumor diameters (cm) & $1.061(1.043-1.079)$ & $<0.001$ \\
AFP value (ng/ml) $>$ 400 & $1.928(1.599-2.325)$ & $<0.001$ \\
Pretransplant treatment & $0.998(0.912-1.091)$ & 0.958 \\
Immunosuppression Induction (reference anti-CD25) & & 0.514 \\
Thymoglobulin & $1.074(0.921-1.253)$ & 0.362 \\
Anti-CD25+Thymoglobulin & $1.370(0.612-3.064)$ & 0.444 \\
Maintenance at discharge & & 0.002 \\
Tacrolimus & & 0.269 \\
Cyclosporine & $0.884(0.819-0.954)$ & 0.108 \\
Sirolimus & $1.127(0.912-1.391)$ & 0.014 \\
Mycophenolate mofetil & $1.157(0.969-1.382)$ & 0.005 \\
Steroids & $0.915(0.852-0.982)$ & $0.898(0.832-0.968)$ \\
\hline AFP
\end{tabular}

AFP, a-fetoprotein; BMI, body mass index; CNS, central nervous system; DCD, donation after cardiac death; HBV, hepatitis B virus; HCV, hepatitis C virus; INR, international normalized ratio; MELD, model for end-stage liver disease; NASH, nonalcoholic steatohepatitis

Table 4 Multivariable analysis of predictors for recipient overall survival

\begin{tabular}{|c|c|c|}
\hline & $\mathrm{HR}(95 \% \mathrm{Cl})$ & $P$ value \\
\hline Donor age (reference Group I) & & $<0.001$ \\
\hline Group ॥ & $1.087(0.979-1.208)$ & 0.119 \\
\hline Group III & $1.124(1.015-1.246)$ & 0.025 \\
\hline Group IV & $1.395(1.215-1.602)$ & $<0.001$ \\
\hline Recipient age & $1.021(1.014-1.027)$ & $<0.001$ \\
\hline Recipient race (reference White) & & 0.013 \\
\hline Black or African American & $1.141(1-1.301)$ & 0.050 \\
\hline Asian & $0.878(0.726-1.062)$ & 0.181 \\
\hline Hispanic/Latino & $0.882(0.783-0.993)$ & 0.038 \\
\hline Other & $1.178(0.85-1.633)$ & 0.326 \\
\hline Underlying liver diseases (reverence HCV) & & 0.011 \\
\hline HBV & $0.724(0.596-0.879)$ & 0.001 \\
\hline Alcoholic liver diseases & $0.935(0.803-1.088)$ & 0.383 \\
\hline $\mathrm{NASH}$ & $0.878(0.748-1.029)$ & 0.108 \\
\hline Other & $0.992(0.864-1.138)$ & 0.904 \\
\hline MELD & $1.013(1.006-1.019)$ & $<0.001$ \\
\hline Recipient serum creatinine (mg/dl) & $1.051(1.008-1.095)$ & 0.019 \\
\hline Sum of tumor diameters $(\mathrm{cm})$ & $1.052(1.033-1.072)$ & $<0.001$ \\
\hline $\operatorname{AFP}(\geq 400 \mathrm{ng} / \mathrm{ml} \mathrm{vs} .<400 \mathrm{ng} / \mathrm{ml})$ & $1.993(1.648-2.412)$ & $<0.001$ \\
\hline Tacrolimus maintenance at discharge & $0.911(0.835-0.994)$ & 0.035 \\
\hline Steroids maintenance at discharge & $0.762(0.696-0.835)$ & $<0.001$ \\
\hline
\end{tabular}

AFP, a-fetoprotein; HBV, hepatitis B virus; $\mathrm{HCV}$, hepatitis C virus; MELD, model for end-stage liver disease; NASH, nonalcoholic steatohepatitis process in HCC patients, which we should not only evaluate the general status of the recipient but also tumor characteristics using HCC selection criteria, such as the Milan Criteria etc. $[8,28]$. We also observed that immunosuppression maintenance with tacrolimus and steroids at discharge were independent predictors. However, the beneficial role of sirolimus on HCC shown in previous literature was not observed in our study [29, 30]. This might be due to the relatively low proportion of sirolimus use in our cohort. Also, we could not evaluate the effect of immunosuppression doses and immunosuppression maintenance duration during the follow-up due to the limited data availability in the current database. Further studies with more detailed information on immunosuppression regime and a larger cohort are needed.

Our study has several limitations. First, as it is based on a large dataset, we could not analyze the possible confounding variables such as tumor biology as assessed by Edmondson-Steiner grade and microvascular invasion, which are important predictors for overall survival and tumor recurrence in $\mathrm{HCC}$ patients. Also, variables related to new therapeutic advances in HCC treatment, such as the use of targeted therapies before or after transplantation, were also not available in the database. This is important because the prognosis of HCC patients has considerably improved in recent years with the development of targeted therapy and immunotherapy. Secondly, as our study included recipients from 2010 to 2017, there was a potential that a relatively short follow-up would 


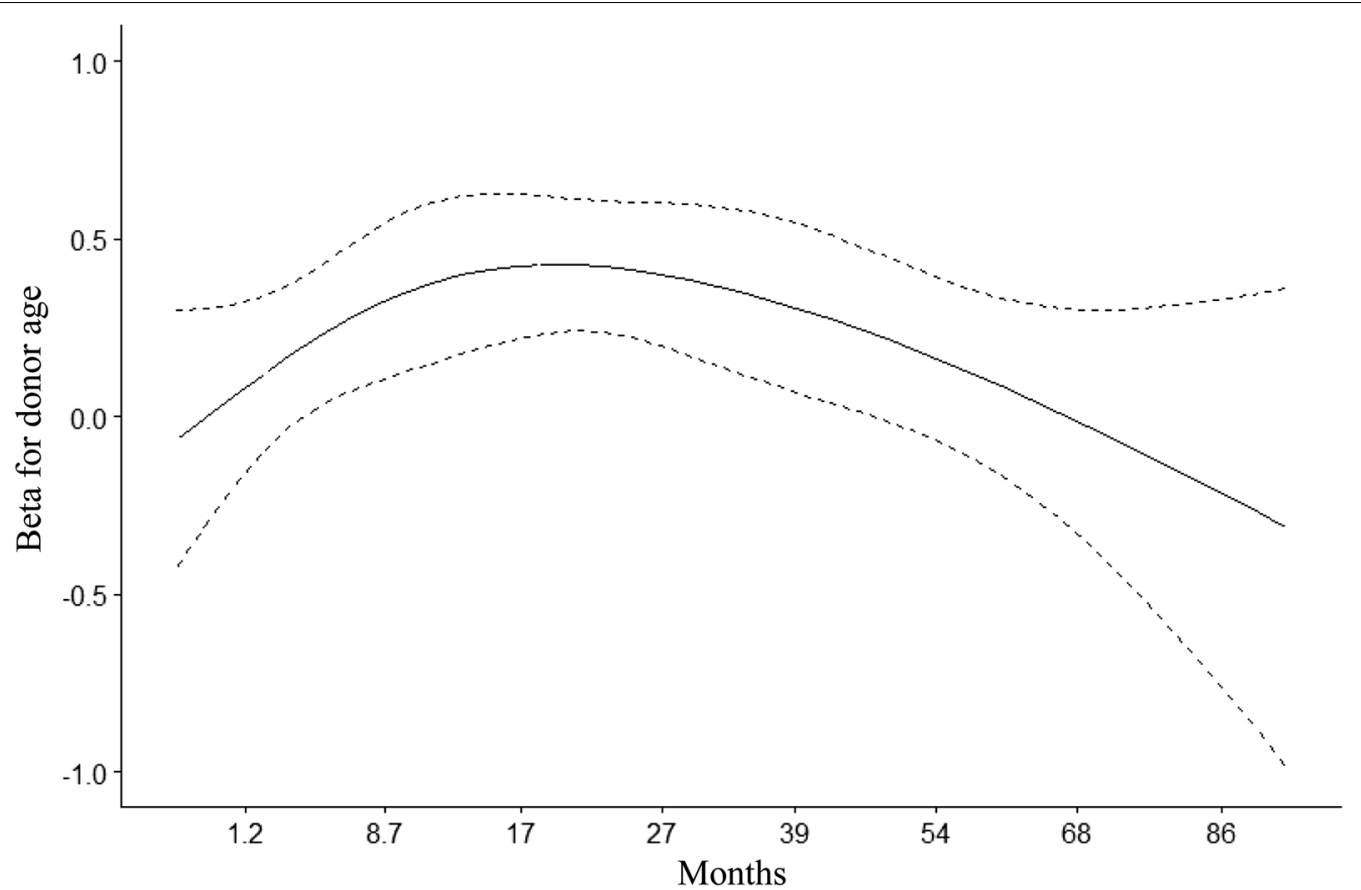

Fig. 4 Cox-derived estimates of the time-dependent hazard ratio of donor age for OS in HCC recipients

confound the true risk of tumor recurrence after transplantation, especially with the currently ongoing development of new treatments for HCC, which might delay or diminish the recurrence of HCC. Nonetheless, our study presents the largest study to date to evaluate the effect of donor age on HCC patients based on the latest transplantation recipient cohort. In the future, prospective studies with long follow-up time need to be designed to investigate the effect of donor age in a more detailed manner to expand the donor pool and benefit more candidates on the waiting list.

\section{Conclusions}

Our study demonstrated that although older donor age was associated with statistical inferior OS in transplant recipients with $\mathrm{HCC}$, the actual survival differences were minimal. Moreover, older donor age was not correlated with decreased liver-specific survival as well as posttransplant tumor recurrence, which might indicate that it had minimal impact on long-term outcomes. For different underlying liver diseases, older donor age was associated with inferior OS in recipients with HCV but with no influence in recipients with $\mathrm{HBV}$, alcoholic liver diseases or NASH. These findings may be useful for clinicians in decision-making with regards to marginal donor allocation and recipient selection to achieve favorable transplant outcomes.

\section{Abbreviations}

AFP: a-Fetoprotein; BMI: Body mass index; Cl: Confidence interval; CNS: Central nervous system; DCD: Donation after cardiac death; DRI: Donor risk index; HBV Hepatitis B virus; HCC: Hepatocellular carcinoma; HCV: Hepatitis C virus; HR: Hazard ratios; INR: International normalized ratio; IRI: Ischemia-reperfusion injury; MELD: Model for end-stage liver disease; NASH: Nonalcoholic steatohepatitis; OPTN: Organ Procurement and Transplantation Network; OS: Overall survival; RFA: Radiofrequency ablation; SRTR: Scientific Registry of Transplant Recipients; TACE: Transarterial chemoembolization.

\section{Supplementary Information}

The online version contains supplementary material available at https://doi. org/10.1186/s12876-021-01786-6.

Additional file 1: Table S1 - S6.

Additional file 2: Figure S1. Overall survival of HCC recipients with posttransplant follow-up time $\geq 24$ months categorized by different donor age groups.

Additional file 3: Figure S2. Liver-specific survival of HCC recipients categorized by different donor age groups.

Additional file 4: Figure S3. Overall survival of HCC recipients with post-transplant follow-up time $\geq 24$ months in different donor age groups according to underlying liver diseases: a HCV; $\mathbf{b} \mathrm{HBV}$; $\mathbf{c}$ alcoholic liver diseases; $\mathbf{d} \mathrm{NASH}$

Additional file 5: Figure S4. Liver-specific survival of HCC recipients in different donor age groups according to underlying liver diseases: $\mathbf{a} \mathrm{HCV}_{\text {; }}$ b HBV; c alcoholic liver diseases; d NASH.

Additional file 6: Figure S5. Overall survival between HCC recipients with and without post-transplant tumor recurrence.

Additional file 7: Figure S6. Cumulative HCC recurrence rates categorized by different donor age groups. 
Additional file 8: Figure S7. Cox-derived estimates of the timedependent hazard ratio of donor age for HCC recurrence after liver transplantation.

\section{Acknowledgement}

The data reported here have been supplied by the Minneapolis Medical Research Foundation, the contractor for the Scientific Registry of Transplant Recipients (SRTR). The interpretation and reporting of these data are the responsibility of the authors and should in no way be seen as an official policy of or interpretation by the SRTR or the U.S. Government.

\section{Authors' contributions}

JZ and ZCH retrieved the associated data from the SRTR database. JZ, ZCH and $Z C$ performed the statistical analysis. JZ wrote the first draft of the manuscript. ZCH, FSX and RLT contributed intellectual content. All authors contributed to critical revisions to the manuscript. SSZ is the corresponding author. All authors approved the final manuscript.

\section{Funding}

This study was sponsored by grants from Innovative Research Groups of National Natural Science Foundation of China (Grant No. 81721091), Research Unit Project of Chinese Academy of Medical Sciences (Grant No. 2019-I2M-5030), Medical Health Science and Technology Project of Health Commission of Zhejiang Province (Grant No. 2020375961). The funders had no role in study design, data collection and analysis, decision to publish, or preparation of the manuscript.

\section{Availability of data and materials}

The data that support the findings of this study are available from the Scientific Registry of Transplant Recipients database, but restrictions apply to the availability of these data, which were used under license for the current study, and so are not publicly available. Data are however available from the authors upon reasonable request and with permission of the Scientific Registry of Transplant Recipients database. The protocol for the present study was in accordance with the Declaration of Helsinki and was approved by the Ethics Committee of the First Affiliated Hospital, College of Medicine, Zhejiang University, China (Approval Number 2019-1020).

\section{Declarations}

\section{Ethics approval and consent to participate}

With Data Release Agreement with the Scientific Registry of Transplant Recipients database, all data in this study were released from SRTR. The protocol for the present study was in accordance to Declaration of Helsinki and was approved by the Ethics Committee of the First Affiliated Hospital, College of Medicine, Zhejiang University, China (Approval Number 2019-1020). And no additional patient consent was required for the measurements in the data analysis.

\section{Consent for publication}

Not applicable.

\section{Competing interests}

The authors declare that they have no competing interests.

\section{Author details}

${ }^{1}$ Division of Hepatobiliary and Pancreatic Surgery, Department of Surgery, First Affiliated Hospital, Zhejiang University, School of Medicine, Hangzhou, China. ${ }^{2} \mathrm{NHC}$ Key Laboratory of Combined Multi-Organ Transplantation, Hangzhou, China. ${ }^{3}$ Key Laboratory of the Diagnosis and Treatment of Organ Transplantation, Research Unit of Collaborative Diagnosis and Treatment For Hepatobiliary and Pancreatic Cancer, Chinese Academy of Medical Sciences (2019RU019), Hangzhou, China. ${ }^{4}$ Key Laboratory of Organ Transplantation, Research Center for Diagnosis and Treatment of Hepatobiliary Diseases, Hangzhou 310003, Zhejiang Province, China.

Received: 28 November 2020 Accepted: 21 April 2021 Published online: 30 April 2021

\section{References}

1. Yang JD, et al. A global view of hepatocellular carcinoma: trends, risk, prevention and management. Nat Rev Gastroenterol Hepatol. 2019;16:589-604.

2. Zucman-Rossi J, Villanueva A, Nault JC, Llovet JM. Genetic landscape and biomarkers of hepatocellular carcinoma. Gastroenterology. 2015;149(1226-1239):e1224.

3. Villanueva A. Hepatocellular carcinoma. N Engl J Med. 2019;380:1450-62.

4. Nardo B, et al. Liver transplantation from donors aged 80 years and over: pushing the limit. Am J Transplant. 2004;4:1139-47.

5. Kan C, Ungelenk L, Lupp A, Dirsch O, Dahmen U. Ischemia-reperfusion injury in aged livers-the energy metabolism, inflammatory response, and autophagy. Transplantation. 2018;102:368-77.

6. Feng $\mathrm{S}$, et al. Characteristics associated with liver graft failure: the concept of a donor risk index. Am J Transplant. 2006;6:783-90.

7. Diaz Jaime F, Berenguer M. Pushing the donor limits: deceased donor liver transplantation using organs from octogenarian donors. Liver Transpl. 2017;23:S22-6.

8. Mazzaferro $V$, et al. Liver transplantation for the treatment of small hepatocellular carcinomas in patients with cirrhosis. N Engl J Med. 1996;334:693-9.

9. Yao FY, et al. Liver transplantation for hepatocellular carcinoma: expansion of the tumor size limits does not adversely impact survival. Hepatology. 2001;33:1394-403.

10. Jay $C$, et al. A comprehensive risk assessment of mortality following donation after cardiac death liver transplant-an analysis of the national registry. J Hepatol. 2011;55:808-13.

11. Orci $L A$, et al. Donor characteristics and risk of hepatocellular carcinoma recurrence after liver transplantation. Br J Surg. 2015;102:1250-7.

12. SRTR. 2020. http://www.srtr.org/.

13. Samoylova ML, Dodge JL, Vittinghoff E, Yao FY, Roberts JP. Validating posttransplant hepatocellular carcinoma recurrence data in the United Network for Organ Sharing database. Liver Transpl. 2013;19:1318-23.

14. Wileyto EP, Li Y, Chen J, Heitjan DF. Assessing the fit of parametric cure models. Biostatistics. 2013;14:340-50.

15. Blok JJ, et al. Validation of the donor risk index in orthotopic liver transplantation within the Eurotransplant region. Liver Transpl. 2012;18:112-9.

16. Braat $A E$, et al. The Eurotransplant donor risk index in liver transplantation: ET-DRI. Am J Transplant. 2012;12:2789-96.

17. Halldorson JB, Bakthavatsalam R, Fix O, Reyes JD, Perkins JD. D-MELD, a simple predictor of post liver transplant mortality for optimization of donor/recipient matching. Am J Transplant. 2009;9:318-26.

18. Li CX, et al. CXCL10/CXCR3 signaling mobilized-regulatory T cells promote liver tumor recurrence after transplantation. J Hepatol. 2016;65:944-52.

19. Ling CC, et al. Post-transplant endothelial progenitor cell mobilization via CXCL10/CXCR3 signaling promotes liver tumor growth. J Hepatol. 2014;60:103-9.

20. Lake JR, et al. Differential effects of donor age in liver transplant recipients infected with hepatitis $B$, hepatitis $C$ and without viral hepatitis. Am J Transplant. 2005;5:549-57.

21. Wong RJ, et al. Nonalcoholic steatohepatitis is the second leading etiology of liver disease among adults awaiting liver transplantation in the United States. Gastroenterology. 2015;148:547-55.

22. Goldberg D, et al. Changes in the prevalence of hepatitis $C$ virus infection, nonalcoholic steatohepatitis, and alcoholic liver disease among patients with cirrhosis or liver failure on the waitlist for liver transplantation. Gastroenterology. 2017;152(1090-1099):e1091.

23. Bilbao I, et al. Our experience in liver transplantation in patients over $65 \mathrm{yr}$ of age. Clin Transplant. 2008;22:82-8.

24. Collins $\mathrm{BH}$, et al. Long-term results of liver transplantation in older patients 60 years of age and older. Transplantation. 2000;70:780-3.

25. Su F, et al. Aging of liver transplant registrants and recipients: trends and impact on waitlist outcomes, post-transplantation outcomes, and transplant-related survival benefit. Gastroenterology. 2016;150:441-53.

26. Sharpton SR, Feng S, Hameed B, Yao F, Lai JC. Combined effects of recipient age and model for end-stage liver disease score on liver transplantation outcomes. Transplantation. 2014;98:557-62.

27. Crespo G, et al. The efficacy of direct anti-HCV drugs improves early postliver transplant survival and induces significant changes in waiting list composition. J Hepatol. 2018;69:11-7. 
28. Xu X, et al. Clinical practice guidelines on liver transplantation for hepatocellular carcinoma in China (2018 edition). Hepatobiliary Pancreat Dis Int. 2019;18:307-12.

29. Kneteman NM, et al. Sirolimus-based immunosuppression for liver transplantation in the presence of extended criteria for hepatocellular carcinoma. Liver Transpl. 2004;10:1301-11.

30. Toso C, Merani S, Bigam DL, Shapiro AM, Kneteman NM. Sirolimusbased immunosuppression is associated with increased survival after liver transplantation for hepatocellular carcinoma. Hepatology. 2010;51:1237-43.

\section{Publisher's Note}

Springer Nature remains neutral with regard to jurisdictional claims in published maps and institutional affiliations.
Ready to submit your research? Choose BMC and benefit from:

- fast, convenient online submission

- thorough peer review by experienced researchers in your field

- rapid publication on acceptance

- support for research data, including large and complex data types

- gold Open Access which fosters wider collaboration and increased citations

- maximum visibility for your research: over 100M website views per year

At BMC, research is always in progress.

Learn more biomedcentral.com/submissions 